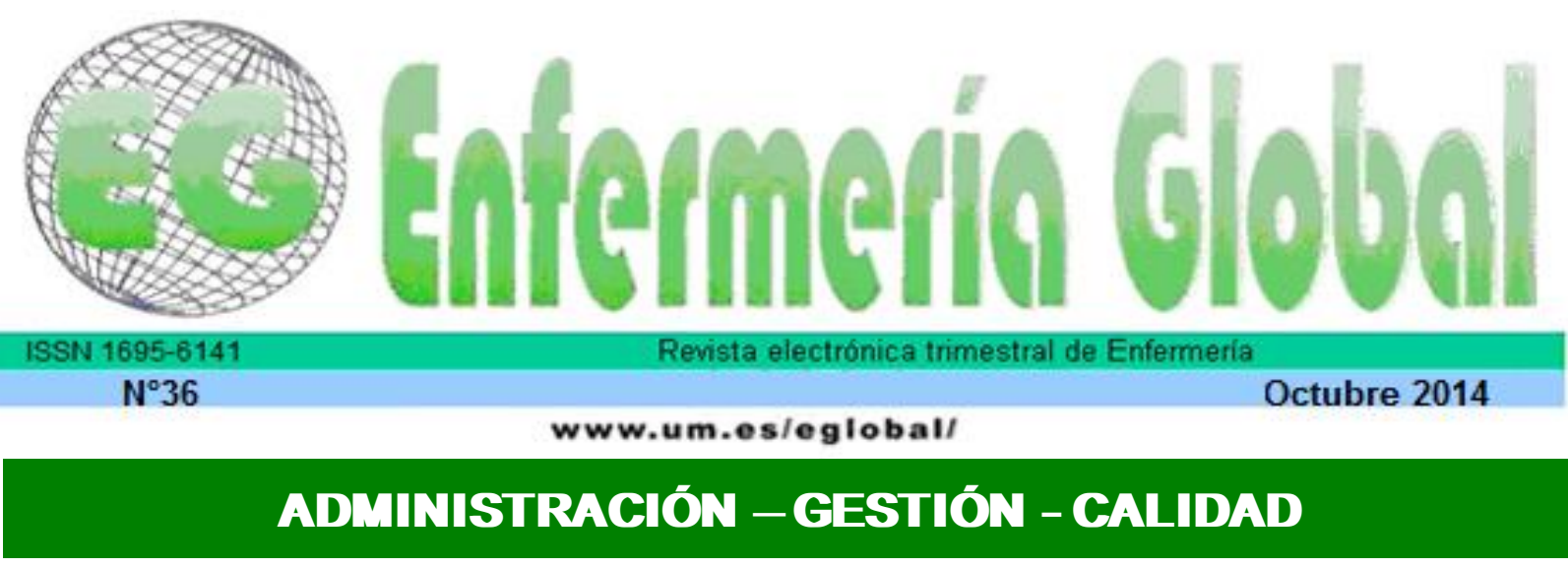

\title{
Trabajo en equipo: visión de los enfermeros de un hospital de Murcia/España
}

Trabalho em equipe: visão de enfermeiros de um hospital de Murcia/Espanha

Teamwork: Vision of nurses in a hospital of Murcia / Spain

*Thofehrn, Maira Buss **López Montesinos, María José ***Amestoy, Simone

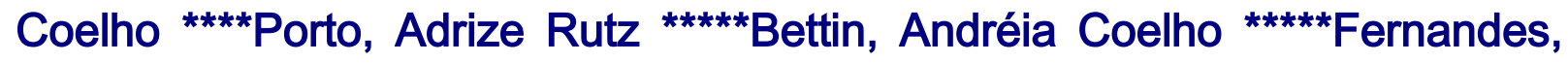
Helen Nicoletti ${ }^{* * \star * *}$ Rodríguez Mondejar, Juan José é ${ }^{* \star * \star * *}$ Mikla, Marzena

* Postdoctora en Enfermería. Profesora Asociada I de la Facultad de Enfermería/UFPel. Coordinadora del Grupo de investigación NEPEN- Núcleo de Estudios en Prácticas de Salud y Enfermería. E-mail: mairabusst@hotmail.com **Doctora en Enfermería. Profesora de la Universidad de Murcia-España ***Doctora en Enfermería. Profesora de la Facultad de Enfermería/UFPel. Miembro del Grupo de investigación NEPEN *** Doctoranda en Enfermería por la Universidad Federal de Rio Grande do Sul. Miembro del Grupo de investigación NEPEN ${ }^{* * * * *}$ Alumna de Máster de Ciencias-ênfasis en Enfermería por el Programa de Posfgraduación en Enfermería/Universidad Federal de Pelotas. Miembro del Grupo de investigación NEPEN ****** Doctorando/a en Enfermería por laa Universidad de Murcia - España.

Palabras clave: Enfermería; equipo de enfermería; ambiente de trabajo

Palavras chave: Enfermagem; Equipe de enfermagem; Ambiente de trabalho.

Keywords: Nursing; Nursing Team; Work environment.

\section{RESUMEN}

Objetivo: Conocer la visión de las enfermeras, que trabajan en un hospital de Murcia, en España, sobre el trabajo en equipo.

Método: Un estudio cualitativo, descriptivo y exploratorio, fue realizado desde noviembre de 2011 a enero de 2012, con 14 enfermeras en el Hospital General Universitario Reina Sofía. La recolección de datos ocurrió a través de dos reuniones de grupos focales. Los datos se analizaron por la propuesta operativa de Minayo.

Resultados: Los enfermeros reconocen que todos los miembros del equipo son importantes y que cada uno aporta sus especificidades. Sin embargo, hay muchas dificultades en el trabajo diario: individualismo, alta rotación de personal, falta de autonomía, relaciones verticales de poder, falta de tiempo para las reuniones y de líderes para construir y fomentar el trabajo en equipo. 
Conclusión: Se destaca que la comunicación efectiva en el lugar de trabajo puede ser capaz de reducir conflictos y promover la formación de vínculos profesionales saludables. Por lo tanto, las actitudes dialógicas son fundamentales para promover el trabajo colectivo

\section{RESUMO}

Objetivo: Conhecer a visão de enfermeiros, que atuam em um Hospital de Múrcia na Espanha, sobre trabalho em equipe.

Método: Trata-se de um estudo qualitativo do tipo descritivo e exploratório, realizado em novembro de 2011 a janeiro de 2012, com 14 enfermeiros que trabalhavam no Hospital Geral Universitário Reina Sofía. A coleta de dados ocorreu por meio de dois encontros de grupo focal. Os dados foram analisados pela proposta operativa de Minayo.

Resultados: os enfermeiros reconhecem que todos os integrantes da equipe são importantes e que cada um contribui com suas especificidades. Entretanto, inúmeras dificuldades são enfrentadas na realização do trabalho cotidiano, como: individualismo, alta rotatividade da equipe, falta de autonomia, relações de poder verticais, falta de momentos de encontros e de líderes para a construção e estímulo do trabalho em equipe.

Conclusão: destaca-se que comunicação efetiva no ambiente de trabalho pode ser capaz de diminuir os conflitos e favorecer a formação de vínculos profissionais saudáveis. Desta forma, posturas dialógicas são fundamentais para promover o trabalho coletivo.

\section{ABSTRACT}

Objective: To know the vision of nurses who work in a Hospital of Murcia in Spain, about teamwork.

Method: This was a qualitative, descriptive and exploratory study, conducted from November 2011 to January 2012, with 14 nurses who worked at the University Hospital Reina Sofía. The data collection occurred through two focus group meetings. Data were analyzed by the proposed operative of Minayo.

Results: The nurses recognize that all team members are important and that each one contributes with its specificities. However, many difficulties are faced in carrying out the daily work, such as individualism, high staff turnover, lack of autonomy, vertical power relations, lack of time for meetings and of leaders to build and to encourage the teamwork.

Conclusion: It is emphasized that effective communication in the workplace may be able to reduce conflicts and promote the formation of healthy professional links. Thus, dialogical attitudes are fundamental to promote collective work.

\section{INTRODUCCIÓN}

El fomento de nuevas formas de comprensión y acción ante las necesidades de salud de la población repercute, cada vez más, en cambios en las prácticas y rutinas laborales que se constituyen en los cuidados ofrecidos a pacientes, familias y comunidades, a fin de promover salud por medio de una postura acogedora, humana y ética de los trabajadores en busca de la integralidad en la asistencia. Por tanto, se hace necesario un mayor entendimento sobre el trabajo de la enfermería.

El proceso de trabajo de los profesionales de enfermería integra el sector de servicios y tiene por finalidad la acción terapéutica de salud; el objeto de trabajo, el individuo enfermo, o grupos saludables, o expuestos a riesgos, que necesitan preservar la salud o prevenir dolencias; como instrumento de trabajo se tienen las herramientas y las conductas que representan el nivel técnico del conocimiento y otros como el relacional, que es el saber de salud. El producto final es la propia prestación de la asistencia que se produce, simultáneamente, al momento en que es consumida. ${ }^{1}$ 
Se destaca que la demanda por cuidado necesita de un trabajo profesional, sea en el enfoque de la promoción de la salud y de la vida, como terapéutico, ejercido de forma individual o coletiva, y en los diversos tipos de instituciones de salud. En este campo de actuación, el cuidado no es práctica exclusiva de la enfermería. El trabajo de otros profesionales de salud puede tener una dimensión cuidadora, sin embargo, es la enfermería la que tiene el cuidado como objeto epistemológico y como centralidad de su actividad profesional. ${ }^{2}$

En este estudio se aborda la característica colectiva del trabajo en la enfermería, o sea, la relevancia del trabajo en equipo, entendido como una herramienta capaz de promover aproximaciones entre los actores involucrados en el proceso salud y enfermedad, además de facilitar la realización de la tarea profesional y contribuir a la satisfacción en el ambiente de trabajo. La idea de equipo deriva de dos sentidos, el de equipo en cuanto agrupamiento de agentes, caracterizado por la fragmentación, y el otro, representando la articulación de las acciones e interacción entre los trabajadores. ${ }^{3}$

Dependiendo de la organización del equipo y de las relaciones que se dan en este espacio, habrá influencias en la asistencia prestada. Cada equipo de enfermería representa un grupo de trabajo único y singular, detentador de una dinámica de relaciones que, en general, se consolida de forma espontánea, generando, muchas veces, conflictos, que si se resuelven mal, pueden hacer que el trabajo se convierta en una jornada de sufrimiento y malestar. De esta forma, investigadores preocupados con la actuación del enfermero, frente a la administración del cuidado y del equipo, construiran un modelo de gestión en enfermería, a fin de potencializar las relaciones interpersonales, esto es, los vínculos profesionales, para alcanzar un cuidado comprometido. ${ }^{4}$

Para acreditar la relevancia del trabajo en equipo, se buscó desvelar nuevas realidades y nuevas posibilidades de ejercerlo de modo a impulsar satisfacción profesional y ampliar la calidad del servicio en toda la red de salud, mediante investigación fuera del país. De esta forma, este estudio tuvo como objetivo conocer la visión de los enfermeros que trabajan en un Hospital de Murcia (España), sobre el trabajo en equipo.

\section{MATERIAL Y MÉTODO}

Se trata de un estudio cualitativo de tipo descriptivo y exploratorio, realizado en el periodo de noviembre de 2011 a enero de 2012. Integraron la investigación 14 enfermeros que trabajaban en el Hospital General Universitario Reina Sofía de Murcia/España, los cuales tenían interés en reflexionar sobre el trabajo en equipo. Esta institución hospitalaria consta de 330 camas, los profesionales ejercían su actividad en tres turnos de trabajo, totalizando 37 horas semanales.

Los datos fueron recogidos por medio de grupos focales. Se realizaron dos encuentros em el propio lugar del estudio, acordados previamente con los participantes. Los datos fueron grabados en audio y transcritos al término de cada encuentro. Con la intención de preservar el anonimato, los mismos fueron identificados por la letra $E$ (Enfermero) seguida de un número ordinal (E1, E2... E14). Para el análisis de los datos se utilizó la propuesta operativa de Minayo, constituida por dos momentos operacionales. El primero consiste en las determinaciones fundamentales del estudio, que se trazan en la fase exploratoria de la investigación. Y 
el segundo momento se denomina de interpretación, pues es el encuentro de las hablas con los hechos empíricos. La fase interpretativa presenta dos etapas: la ordenación de los datos y la clasificación de los datos, esta última incluye la lectura horizontal y exhaustiva de los textos, lectura transversal, análisis final y la construcción del informe con la presentación de los resultados. ${ }^{5}$

Para el desarrollo de la investigación se respetaron todas las prerrogativas éticas previstas en la Resolución n‥ 196/96 sustituída por la Resolución №. 466/2012 del Ministerio de Salud, que trata de la investigación involucrando a seres humanos, siendo aprobada por el Comité de Ética en Investigación del Hospital Santa Casa de

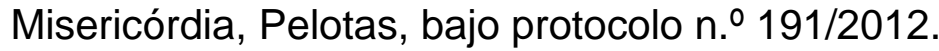

\section{RESULTADOS Y DISCUSIÓN}

A partir del análisis de los datos emergieron tres temas: visión de los enfermeros sobre trabajo en equipo; dificultades vivenciadas por los enfermeros para trabajar en equipo y estrategias para el trabajo en equipo: atributos de la comunicación efectiva; los cuales serán presentados en la secuencia.

\section{Visión de los enfermeros sobre el trabajo en equipo}

Los relatos de los enfermeros, integrantes del estudio, muestran una concepción del trabajo en equipo marcado por la percepción de algo complejo de ser realizado. Además de esto, abordaron la distinción entre grupo y equipo, así como el compromiso de los profesionales para el alcance de los objetivos comunes al equipo, el establecimiento de relaciones de trabajo fundamentadas en el intercambio de saberes y en las relaciones horizontales.

La mención de la complejidad del trabajo en equipo aparece en la siguiente declaración:

[...] el trabajo en equipo, especialmente era como algo casi exclusivo, la única forma de trabajar, y relativamente fácil, trabajar en equipo. Que con el paso del tiempo es más complejo. (E1)

Se percibe en el relato mencionado que el trabajo en equipo es visto bajo una mirada incipiente y concebido como algo simple de desempeñar, por eso en el transcurso del proceso surgen las dificultades. Estas pueden estar relacionadas con las vivencias personales y profesionales que van a determinar e influir en el proceso de formación de los equipos. ${ }^{6}$ Por ello, resulta imprescindible valorar la subjetividad de las relaciones interpersonales para el fortalecimiento del trabajo en equipo. ${ }^{7}$

La diferenciación entre equipo y grupo emerge en el testimonio:

Una cosa es trabajar en grupo, otra cosa es trabajar en equipo. No yo, el ejemplo que yo pongo a los alumnos, por ejemplo, es que como somos todos un grupo, cuando digo que vamos a trabajar, se hace equipo de cuatro. (E2) 
El relato apunta la percepción por parte de uno de los enfermeros entre los conceptos de equipo y grupo. La declaración coloca como grupo un número de personas que al desempeñar una tarea se convierte en un equipo, contraponiendose a esta definción tenemos el referencial pichoniano que define equipo como un grupo de personas ligadas por constante de espacio y tiempo que se proponen desempeñar una tarea común. $^{8}$

En este sentido, otros aspectos importantes destacados por los profesionales indican como elementos constituyentes para la efectivación del trabajo en equipo la determinación de objetivos a ser alcanzados por el grupo:

Temos preestabelecido adonde va el equipo, los productos que queremos hacer. Poco a poco, tenemos una concepción de cuál es nuestro nivel, digamos de calidad de asistencia, que [...] no tiene un patrón, digamos de equipo, o no tenemos consenso, no hablo de la exigencia médica, medicación que se hace de servicios mínimos, mas consideramos un equipo que nosotros estamos trabajando bien y que es a lo que llegamos. No me refiero al nivel técnico, sino a todos los niveles del equipo. (E3)

Se observa en el testimonio la importancia del establecimiento de metas y objetivos deseados por el equipo para el alcance de la calidad asistencial ofrecida a los enfermos del servicio. Se destaca que no hay grupo sin tarea y el establecimiento del objetivo del grupo consiste en la tarea que permite la elaboración y resolución de las ansiedades relacionadas con el objeto de conocimiento, favoreciendo la correcta lectura de la realidad y garantía de eficiente proceso de aprendizaje, por tanto la tarea es uno de los principales organizadores de la estructura del grupo. ${ }^{8}$

El intercambio de saberes emerge en el siguiente testimonio como elemento para el trabajo en equipo:

Yo estoy recordando, cuando el hospital abrió vinieron médicos que hacían sesiones clínicas. Porque los médicos tienen muy claro el concepto de trabajo en equipo [...] fui invitada a entrar en una de esas sesiones y fue extraordinario. Porque todo lo que se hacía, se hacía con la enfermería. Yo llevaba toda la información que tenía de estas historias, de estos pacientes y de este dia. Asi que tenía un equipo [...] porque ellos llevaban sus prescripciones, y nosotros llevabamos la nuestra. (E2)

De hecho un profesional que ya desempeña un servicio, una actividad concreta por muchos años, transmite seguridad a los médicos [...] usted hace una evaluación, como diciendo, mi trabajo es válido, confien en mí. Mas tenemos que ganar día a día [...] el médico cuando sale (de la facultad) parece que gana todo pronto y tiene su lugar establecido $y$, nosotros tenemos que ir ganando día a día, pero ¿por qué? ¿Por qué nosotros nos consideramos inferiores? [...] tienen lugares que tal vez [...] mas debería existir una relación más adecuada, igual, pero no existe [...] existen grupos muy cerrados, que no quieren ver la necesidad de la interdisciplinaridad [...] y todos formamos una 
cadena, así, yo lo veo como una cosa muy importante para el futuro. (E8)

Los testimonio abordan la importancia de que profesionales de especialidades diferentes compartan sus saberes, a pesar de estar enfatizando cómo los profesionales del área médica se colocan en posición de superioridad. Algunas profesiones debido a la legitimación social o por valorización de los demás profesionales son colocados em destaque. ${ }^{3}$ Por eso para un trabajo en equipo los profesionales de diversas áreas del conocimiento deben partilhar los saberes en una relación de igualdad para la construcción colectiva de un plan terapéutico específico para cada paciente, respetando su individualidad.

Para ello, algunos participantes del estudio señalan como factores determinantes para establecimento de equipo de trabajo la horizontalidad de las relaciones en el trabajo:

Yo no trabajo mejor que nadie. Porque cada uno tiene su manera de hacer las cosas. Es respetable. Entonces, si fuese lo mejor, estaría trabajando en los tres turnos. (E4)

Unos tienen más contacto en el día a día, otros menos, pero al final, nos necesitamos todos. Así que tiene que haber personas que trabajen en equipo [...] que sean sensibles, que no les importe pedir ayuda. (E1)

Los testimonios señalan el reconocimiento por parte de los profesionales de que todos los integrantes del equipo son importantes y que cada uno contribuye con sus especificidades, así como la relevancia en establecer relaciones recíprocas entre los profesionales para la prestación del cuidado. En esta perspectiva, la integración entre los miembros del equipo es vista como un proceso social que tiene como objetivo unificar al equipo, por la inclusión de todos los miembros sin descuidar las particularidades de cada uno, así a partir del instante en que ocurre la participación de las personas entre sí en la ejecución del trabajo, hay el establecimiento de la cooperación. ${ }^{9}$

El establecimiento de relaciones de igualdad y corresponsabilización contribuye al favorecimiento de una nueva dimensión de trabajo, proporcionando calidad al cuidado prestado. ${ }^{6}$ Un estudio realizado sobre satisfacción en el trabajo mostró que la falta de integración y de compromiso profesional entre los colegas del equipo de enfermería son factores generadores de insatisfacción en el trabajo. ${ }^{10}$

De esta forma, se percibe, en la presente investigación, que los enfermeros consideran el trabajo en equipo esencial para el desarrollo individual y colectivo, el cual, si construído efectivamente, puede repercutir de modo positivo en la producción de un cuidado cualificado.

\section{Dificultades vivenciadas por los enfermeros para trabajar en equipo}

Conforme los resultados, se destacan las siguientes dificultades vivenciadas por los enfermeros para trabajar en equipo: individualismo, falta de autonomía, rotatividad, necesidad de enfermeros-líderes actuando en el ambiente hospitalario. 
El individualismo fue el principal obstáculo, apuntado por los enfermeros, para un efectivo trabajo en equipo. Las opiniones que siguen representan un fragmento de este resultado:

El problema es el individualismo de la profesión. Yo creo que es la causa más extrema de que nosotros no avancemos como profesión. (E4)

Es que desde el colegio, comienzan a educar el individualismo [...] y no se enseña a colaborar. $Y$ esto continúa en la universidad y después usted percibe que no se trabaja solo, que se trabaja en grupo. (E3)

Cabe aquí mencionar que el individualismo está presente en nuestra sociedad globalizada, en virtud de los nuevos valores desencadenados a partir de esta realidad, donde presenciamos relaciones extremadamente superficiales, que son sustituídas de la misma forma que cambiamos de vestido. Tal fenómeno de fluidez en las relaciones, causa inseguridad, determinando el aislamiento de los seres humanos, ${ }^{11}$ además de llevar a una situación de destrucción y crueldad en las relaciones sociales. ${ }^{12}$

Ante esto, el individualismo acaba por perjudicar el rendimiento, las relaciones interpersonales y el clima organizacional. Con todo, cabe resaltar que la formación profesional y las exigencias político-sociales impuestas por el mercado de trabajo, exaltan a su vez esta característica. Por esto, la educación de enseñanza superior deberá repensar los modos de formación, a fin de permitir el desarrollo de competencias y habilidades dirigidas a la necesidad de los profesionales de actuar colectivamente.

Existe la necesidad de promoción del trabajo colectivo, uniendo sus atribuciones individuales positivas para obtener propósitos en común, formando equipos, potenciando su desempeño y alcanzando resultados además de los esperados. ${ }^{13}$

También, interfiriendo de modo negativo en el trabajo en equipo emergió en el estudio la falta de autonomía:

Si usted es coordinador, es alguien superior a mí, es quien decide lo que es bueno para mí. Mas también tiene que cuidar del paciente, en el trabajo, en nuestra unidad. O sea, si mi jefe me dice: [...] cuida este paciente de esta manera, el plan de cuidado es sólo eso. No me permite desarrollar. (E5)

De modo sorprendente, la falta de autonomía, emerge en los resultados, como es habitual, vuelta a la organización histórica del trabajo, en que la medicina mantiene su papel destacado en la conducción de la asistencia. Pero se refiere, en cierta forma, a la reducción de la creatividad impuesta por el enfermero supervisor sobre las acciones de los enfermeros de cabecera. Se identifica a partir del testimonio de que el enfermero supervisor adopta una postura centralizadora, con poca flexibilidad y apertura para el diálogo, evidenciando las relaciones de poder y mando.

El poder visto de modo reduccionista, en el sentido de comando, puede dificultar la creatividad de la persona que sufre el proceso, desencadenando una fuerza coercitiva que puede llevar a la violencia. Para romper este ciclo de sufrimiento y establecer 
vínculos profesionales saludables en el equipo, el poder debe ser ejercido de modo democrático y participativo, con respeto a todos los participantes que forman el grupo de trabajo, además del respeto por el desarrollo del proyecto de cuidado delineado y aprobado por el propio equipo. ${ }^{4}$

En los testimonios que siguen se perciben las incoherencias, las pausas, las dificultades para tornar verbal cómo ocurren las relaciones con el equipo médico y como estas relaciones aún son tenues.

Un día tuve que batirme de frente con un médico y le dije, literalmente: "yo soy un profesional de esto", o sea, nosotros tenemos que hacer valer lo nuestro [...] el desafio que tenemos es este: buscar nuestro lugar y hacernos de respetar por los demás. (E8)

Así que a veces hay personas... (pausa)... concretas... de otros tipos de... y, estamos hablando de médicos. Es... se hace trabajo en equipo. Entre una y otra... trabajar en equipo, mas pocas veces. Esta es mi experiencia. Pero es así... no acordamos las acciones. No existe un acuerdo de objetivos con un paciente... para mí no tiene equipo. (E6)

La autonomía representa la maduración del ser para sí, de esta forma, consiste en un proceso, es llegar a ser autónomo, no teniendo fecha marcada para acontecer. Así, ninguno es autónomo primero para después decidir. La autonomía se constituye en varias decisiones que van siendo tomadas. Por tanto, su aprendizaje debe estar centrado en experiencias estimuladoras de la decisión y de la responsabilidad, así como respetuosas y promotoras de libertad. ${ }^{14}$

La falta de autonomía también fue relatada en relación al paciente, o sea, el individuo hospitalizado puede ser despersonificado por el equipo. De ese modo, los profesionales pueden supervalorizar su conocimiento técnico-científico frente al saber del enfermo y colocarlo en una condición de pasividad, con mínimo espacio para tomar decisiones sobre su propia vida, tornándolo de su propriedad:

Si yo me hago dueña del paciente. En el sentido de que, como yo sé cuidarte, usted calla y me escucha, come sin sal, lava sus dientes tres veces al día y el curativo tiene que cambiar ahora mismo. O sea, si yo no acepto, no escucho al paciente, no le trato al mismo nivel que yo, también puedo afectar al cuidado. Que yo soy especialista en el cuidado del paciente. (E5)

En su declaración, el participante describe algunas prácticas autoritarias y prescriptivas que ocurren entre profesionales y pacientes, se percibe el poder sobre el cuerpo de la persona hospitalizada, pudiendo esta ser asistida como objeto pasivo, libre de opciones y de conocimientos previos, ya que el conocimiento técnicocientífico posee gran sustentación y valorización en el ambiente hospitalario, estando enfocado en el biologicismo y en la práctica curativa. Por otro lado, existe la preocupación del participante en cualificar su cuidado prestado, a través de la escucha, aceptación y respeto a la persona enferma. 
En el ámbito brasileño todavía se dan práticas autoritarias semejantes, pero gracias al contrato de los profesionales de la salud, gestores y pacientes, en busca del fortalecimiento de prácticas para la promoción de la salud, buscando la superación de iniquidades, se ven posibilidades de distanciamiento de prácticas opresoras, veladas, con poco espacio para el diálogo, autonomía y corresponsabilización de los sujetos sobre su proceso de cuidado.

La rotatividad fue otro aspecto elencado por los participantes como obstáculo para el trabajo en equipo:

Porque, yo puedo trabajar bien con mi compañera, pero dentro de un mes tal vez ella ya no esté, y me traen otra persona a la que yo me tengo que adaptar. Y dentro de dos meses, esa persona, u otra compañera, no está más. Esto tampoco es forma de crear un equipo de trabajo. (E6)

Incluso habiendo sido desarrollado en la realidad de Murcia, resultado semejante fue encontrado en un estudio en Brasil, en el que participaron enfermeras que ejercen su práctica profesional en el ámbito hospitalario, que incluyeron la rotatividad como una característica institucional que perjudica la realización de las actividades y la consolidación del equipo de enfermería. ${ }^{15}$

En el escenario hospitalario, la rotatividad de los trabajadores de salud todavía representa un problema enfrentado por los gerentes de enfermería que interfiere de forma significativa en la calidad del cuidado y también en los costos para la organización. Además, esta situación genera insatisfacción del equipo de enfermería, en virtud de la sobrecarga de trabajo. ${ }^{16}$ La intensa rotatividad, igualmente interfiere en la formación continua de un equipo de trabajo cohesionado y de vínculos saludables.

También emergió en el estudio la necesidad de que los enfermeros ejerzan la función de líderes, a fin de administrar el cuidado y el trabajo en equipo.

Y después, si no tenemos una persona líder que nos diga: el objetivo es este. [...] vuelta a decir lo mismo, hay gestores de recursos, de materiales, no hay gestores de equipos de trabajo. Personas son personas, y eso necesita de gerenciamento. (E7)

Pero la función administrativa consume mucho nuestro tiempo [...] porque todo se hace por código, con acceso, que sólo usted (enfermero) tiene, en todos los servicios. (E1)

En las declaraciones emerge el distanciamiento del enfermero en cuanto a las cuestiones de liderazgo de las relaciones humanas entre los trabajadores del equipo, lo que puede tener interferencia en la calidad de vida de los mismos.

De esta forma, cabe mencionar que el liderazgo se caracteriza como una competencia profesional, la cual representa capacidad del enfermero-líder en influir en las personas, mediante el ejercicio del diálogo, buscando alcanzar los objetivos establecidos por el grupo, que en el caso de la enfermería se refiere al cuidado. ${ }^{17} \mathrm{Se}$ complementa que liderar consiste en la capacidad del líder de conducir y administrar el trabajo del equipo, con la intención de realizar una atención eficiente, norteado por conocimientos técnico-científicos y por el dominio de habilidades y competencias 
humanas e interpersonales. $^{18}$

Sin embargo, liderar no es tarea fácil, incluso porque el enfermero, en cuanto líder del equipo de enfermería administra diariamente conflictos y recursos materiales, lo que muchas veces lo distancia de esta responsabilidad. Ante esto, destaca la propuesta de un liderazgo en la perspectiva dialógica, que consiste em la competencia del líder de influir sus colaboradores a actuar de modo crítico y reflexivo sobre su praxis, por medio de la promoción de un proceso de comunicación eficiente. Representa, también la construcción de relaciones horizontales en el ambiente de trabajo, en que florecen oportunidades de socialización de conocimientos y mejora colectivas, habiendo espacio para autonomía y participación activa de los profesionales de salud y pacientes em los procesos decisorios. ${ }^{17}$

\section{Estrategias para el trabajo en equipo: permeadas por la comunicación efectiva}

Los enfermeros mencionaron estrategias para el trabajo en equipo, entre ellas la relevancia de planear la asistencia en conjunto, de trabajar de ese modo y de tener momentos para reuniones:

Creo que siempre tiene que haber un grupo de profesionales que se junten. Los que están trabajando no pueden dejar de trabajar para juntarse. El espacio para reunirnos no existe. Todos trabajamos y esto es trabajo en equipo, que proporciona a cada uno, complementarse. (E7)

Yo pienso que el coordinador tiene la función de facilitar las herramientas para que nosotros mismos nos demos cuenta de que es necesario trabajar en equipo. Yo veo que tenemos que reunirnos $y$ de esta manera intercambiar conocimientos, intercambiar opiniones, tal vez su manera de cuidar complemente el de otra enfermera. Y lo que ella hable, te puede parecer mejor, y de ahí tu cambio su forma de actua, por ejemplo. (E4)

En los testimomios queda demostrado que los encuentros entre los integrantes del equipo son esenciales para el intercambio de informaciones sobre las necesidades de salud de los pacientes. Así como realizar el plan común de la asistencia de enfermería para que cada profesional pueda actuar de manera conjunta y administrar el cuidado de forma eficaz.

En ese sentido, se evidencia que la comunicación, además de la transmisión de informaciones, incluye diálogo como el principal instrumento para la promoción del trabajo en equipo, siendo que el recurso más utilizado para propiciarlo son las reuniones, en distintas modalidades, como la discusión de caso clínico. Para que las reuniones del servicio se concreticen se precisan aspectos estructurales como posibilidad de encuentro de los profesionales de diferentes turnos de trabajo, espacio físico adecuado, disponibilidad de tiempo de los profesionales, comunicación efectiva. Además, las reuniones deben estar dirigidas para la finalidad del trabajo en salud y ser un momento para manifestación, no sólo de las necesidades de salud de los pacientes y profesionales, sino también de los problemas e insatisfacciones del equipo. $^{19}$ 
Otra estrategia para el trabajo en equipo está también relacionada con el diálogo eficaz, pues el feedback entre los profesionales posibilita el desempeño efectivo del equipo:

El feedback de manera casi atropellada y muy informal. O sea, yo descubro lo que puede incomodar a los colegas, puede ser la enfermera, llevando un antibiótico y colocar de una forma. $A$ veces, oigo ya el nivel de protesta, cuando la enfermera, o dos o tres, ya están con rabia de lo que está aconteciendo, y me dicen: "¿Ustedes no dijeron a ella? ¡Nosotros hablamos, claro!" Ese feedback es tan informal, atropellado y casual, pues se tiene la sensación, algunas veces, de un piano un tanto desafinado. Así sería, tal vez, una manera de comenzar, de posibilitar estos pequeños grupos, en que se tenía un feedback, inclusive sentimientos dentro del trabajo, porque pasamos siete horas seguidas con las personas, sin saber lo que sienten mis colegas, pero no voy a saber, alguien me comenta y acabo conociendo por casualidad. (E8)

Los problemas de comunicacióno son los principales responsables de gran parte de los errores operacionales, desaciertos de gestión y conflictos interpersonales. EI alcance del objetivo de la comunicación puede ocurrir por medio de los procesos de retorno del mensaje, esto es, 'feedback' y escucha activa, teniendo especial relevancia en el equipo de enfermería para el desempeño eficaz en las diversas acciones del cuidado al paciente. ${ }^{20}$

Igualmente articuladas a los atributos de la comunicación efectiva están la humildad y las relaciones de poder horizontales en el trabajo, así como la autonomía del equipo para planear y tomar sus decisiones. Estas estrategias son fundamentales para trabajar en equipo y están expuestas en las siguientes declaraciones:

Tiene que ser humilde. Tiene que pedir ayuda y saber trabajar en equipo. Y trabajar en equipo es opuesto de decir que está por encima de los demás, porque soy enfermera y usted es auxiliar. Usted hace un trabajo de engranaje. (E8)

Estuve junto a las otras personas en la $\mathrm{UCl}$ por lo menos tres años sin ningún supervisor. $Y$ trabajamos en equipo perfectamente. Coordinabamos, cuando había problema y tal, decíamos: están bajando, yo encontré esto mal, o ese cuarto está mal. Hacíamos reuniones. Gestionabamos como equipo. (E7)

En este testimonio se destaca que la disponibilidad para el diálogo y socio puede ser traducida en la virtud de la humildad, presupuesto básico de la labor interdisciplinar, dado que un trabajador humilde reconoce los límites y tiene comportamiento activo de insatisfacción y busca. ${ }^{21}$

Del mismo modo, la relación jerárquica menos burocratizada y más horizontalizada, personalizada y dialogada es característica del trabajo colectivo. ${ }^{22}$ Pues, frente a los aspectos contrarios, un trabajador, valiéndose de su poder jerárquico, puede asediar moralmente a otro miembro del equipo, como en situaciones en que los jefes asumen posturas autoritarias y arrogantes en los contactos interpersonales. ${ }^{23}$ 
La perspectiva mencionada necesita ser reflexionada en y con el equipo de enfermería, que está compuesto por diferentes cargos tanto en la realidad brasileña, como en la española. En la institución estudiada existen: enfermeros, con formación superior, subdivididos en enfermeros supervisores responsables de los equipos de enfermería de los diversos sectores del hospital y enfermeros de cabecera; también, auxiliares con formación mediante cursos de capacitación, estando bajo la supervisión del enfermero de cabecera. Esta realidad jerárquica está asociada a la posición que cada componente del equipo ocupa en el servicio y a su lugar en la división técnica y social del trabajo. Aunque esta división del trabajo produzca fragmentación de los cuidados de enfermería, en el trabajo en equipo debe haber interdependencia y complementaridad del hacer de los profesionales. ${ }^{24}$

También asociada a la interdependencia de los profesionales está el equipo autónomo cuando requiere atribuciones y responsabilidades de cada trabajador, elaboración del modo de cobro y prestación de cuentas en relación a los resultados. ${ }^{19}$

No obstante, el grupo de enfermeros resalta la investigación y el perfeccionamiento profesional como fuente de motivación y ascenso profesional para los trabajadores, así como la estrategia de abordaje transversal del asunto trabajo en equipo, desde la enseñanza básica y para los multiprofesionales:

Hace 20 años, nunca habríamos conseguido un grupo focal en este hospital. No porque fuesemos peor, y me incluyo, sino porque había una motivación muy diferente. Esto está favoreciendo mucho la motivación. Las personas ahora quieren mejorar. Buscan calidad. Buscan de alguna manera, como esta investigación que estás haciendo, mejorar el sistema educativo. Entonces, esto, es el nivel académico que los profesionales de enfermería (bachillerato desde 2009, anterior a esta fecha eran tecnicos) tienen hoy día, está mejorando mucho. (E2)

Entonces yo pienso que desde la formación, debería facilitarse muchísimo la relación, dar énfasis al trabajo en equipo, mas, no sólo en la enfermería, en la enseñanza media, auxiliares, $y$ también en la enseñanza médica. (E4)

Las declaraciones reflejan como la transformación de la realidad es potenciada por la educación permanente en salud, además de ser un fortalecedor del trabajo en equipo. Pues la educación es un proceso de autorrealización y su éxito depende del empeño de todos los sujetos involucrados en los nuevos descubrimientos. ${ }^{21}$

Otro estudio obtuvo como resultados que la educación permanente está relacionada con el interés personal por el aprendizado, con la integración, con el trabajo en equipo y con la responsabilidad con la comunidad. ${ }^{25}$ Por tanto, el marchamo 'trabajo en equipo' precisa estar presente en los currículos desde la enseñanza básica, pues abarca diversos atributos relacionados con la comunicación efectiva, que requiere tiempo para el desarrollo de competencias y habilidades para actuar colectivamente en enfermería. 


\section{CONSIDERACIONES FINALES}

La investigación permitió conocer la visión de los enfermeros del Hospital General Universitario Reina Sofía de Murcia/España en relación al trabajo en equipo. Los hallazgos trazan el reconocimiento por parte de los profesionales de que todos los integrantes del equipo son importantes y que cada uno contribuye con sus especificidades. Sin embargo, evidencian muchas dificultades enfrentadas en la realización del trabajo cotidiano, las cuales interfieren significativamente en la actuación de los equipos como el individualismo, la alta rotatividad del equipo, la falta de autonomía, las relaciones de poder verticales, la falta de momentos de encuentro y de líderes para la construcción y estímulo del trabajo en equipo.

Las estrategias encontradas para minimizar estas dificultadades están asociadas a la comunicación efectiva. En este sentido, se evidencia que la comunicación transmite informaciones e incluye el diálogo como el principal instrumento para la formación de un equipo con vínculos profesionales saludables.

Resulta evidente que la comunicación efectiva en el ambiente de trabajo puede ser capaz de evitar problemas operacionales, de gestión y conflictos interpersonales. También se destaca en este estudio que la virtud de la humildad y la relación jerárquica menos burocratizada y más dialogada son fundamentales para la relación saludable y puede ser un factor determinante para el trabajo colectivo.

Esta investigación muestra su relevancia al trazar la experiencia de trabajadores de enfermería de Murcia/España, para los que las vivencias cotidianas, las dificultades y las potencialidades del trabajo en equipo son muy próximas a las brasileñas, a pesar de las diferencias sociales, económicas y culturales. Sin embargo, se espera que esta investigación pueda contribuir para enfermería señalando que es posible el trabajo en equipo, siendo que para ello se hace necesario reconocer las dificultades personales, grupales e institucionales para buscar estrategias para sanarlas.

\section{REFERENCIAS}

1. Pires D. Reestruturação produtiva e trabalho em saúde no Brasil. 2. ed. São Paulo: Confederação Nacional dos Trabalhadores; Annablume; 2008.

2. Pires D. A enfermagem enquanto disciplina, profissão e trabalho. Rev bras enferm. [on line]. 2009 [citado 12 fevereiro 2013];62(5):739-44. Disponível em: http://www.scielo.br/pdf/reben/v62n5/17.pdf

3. Peduzzi M. Equipe multiprofissional de saúde: conceito e tipologia. Rev saúde pública. [on line]. 2001 [citado 12 fevereiro 2013];35(1):103-9. Disponível em: http://www.scielo.br/pdf/rsp/v35n1/4144.pdf

4. Thofehrn MB, Leopardi MT. Teoria dos Vínculos Profissionais: um novo modo de gestão em enfermagem. Texto \& contexto enferm. [on line]. 2006 [citado 12 fevereiro 2013];15(3):409-17. Disponível em: http://www.scielo.br/pdf/tce/v15n3/v15n3a04.pdf

5. Minayo MCS. O desafio do conhecimento.12. ed. São Paulo: Hucitec; 2010.

6. Araújo MBS, Rocha PM. Trabalho em equipe: um desafio para a consolidação da estratégia de saúde da família. Ciênc saúde coletiva. [on line]. 2007 [citado 12 fevereiro 2013];12(2):455-64. Disponível em: http://www.scielo.br/pdf/csc/v12n2/a22v12n2.pdf

7. Thofehrn MB, Amestoy SC, Porto AR, Arrieira ICO, Dal Pai D. A dimensão da subjetividade no processo de trabalho da enfermagem. Rev enferm saúde. [on line]. 
2011 [citado 12 fevereiro 2013];1(1):190-8. Disponível em: http://www.ufpel.edu.br/revistas/index.php/enfermagemesaude/article/viewFile/58/43

8. Pichon-Rivière E. O Processo Grupal. 8 ed. São Paulo: Editora WMF Martins Flores; 2009.

9. Thofehrn MB, Leopardi MT. Teoria dos Vínculos Profissionais: formação de um grupo de trabalho. Pelotas: Editora Universidade Federal de Pelotas; 2009.

10. Melo MB, Barbosa MA, Souza PR. Satisfação no trabalho da equipe de enfermagem: revisão integrativa. Rev latinoam enferm. [on line]. 2011 [citado 12 fevereiro 2013];19(4):[09 telas]. Disponível [cm: http://www.scielo.br/pdf/rlae/v19n4/pt_26.pdf

11. Pivetta DMP, Matos LS, Alexandre IJ. Crise de identidade do sujeito. Revista Eventos Pedagógicos. Maio-jul, 2012; 3(2):337-45 .

12. Vares SF. Socialismo e indivialismo em Émile Durkheim. Caderno CRH. Maio-ago, 2011; 24(62):435-46.

13. Amestoy SC, Cestari ME, Thofehrn MB, Milbrath VM. Características que interferem na construção do enfermeiro-líder. Acta paul enferm. [on line]. 2009 [citado 12 fevereiro 2013];2(5):673-8. Disponível em: http://www.scielo.br/pdf/ape/v22n5/12.pdf

14. Freire P. Pedagogia da autonomia: saberes necessários à prática educativa. São Paulo: Paz e Terra; 1996.

15. Amestoy SC, Cestari ME, Thofehrn MB, Milbrath VM, Porto AR. Características institucionais que interferem na liderança do enfermeiro. Rev gaúch enferm. [on line]. 2009 [citado 12 fevereiro 2013];30(2):214-20. Disponível em: http://seer.ufrgs.br/RevistaGauchadeEnfermagem/article/view/7723/6678

16. Nomura FH, Gaidzinski RR. Rotatividade da equipe de enfermagem: estudo em hospital-escola. Rev latinoam enferm. [on line]. 2005 [citado 12 fevereiro 2013];13(5):648-53. Disponível em: http://www.scielo.br/pdf/rlae/v13n5/v13n5a07.pdf

17. Amestoy SC. Liderança dialógica: perspectivas na formação de enfermeiroslíderes [tese]. Florianópolis (SC): Programa de Pós-Graduação em Enfermagem, Florianópolis; 2012.

18. Strapasson MR, Medeiros CRG. Liderança transformacional na enfermagem. Rev bras enferm. [on line]. 2009 [citado 12 fevereiro 2013];62(2):228-33. Disponível em: http://www.scielo.br/pdf/reben/v62n2/a09v62n2.pdf

19. Peduzzi M, Carvalho BG, Mandú ENT, Souza GC, Silva JAM. Trabalho em equipe na perspectiva da gerência de serviços de saúde: instrumentos para a construção da prática interprofissional. Physis (Rio J.). [on line]. 2011 [citado 12 fevereiro 2013];21(2):629-46.

Disponível

http://www.scielo.br/pdf/physis/v21n2/a15v21n2.pdf

20. Broca PV, Ferreira MA. Equipe de enfermagem e comunicação: contribuições para o cuidado de enfermagem. Rev bras enferm. [on line]. 2012 [citado 12 fevereiro 2013];65(1):97-103. Disponível em: http://www.scielo.br/pdf/reben/v65n1/14.pdf

21. Haas CM, Ninica DP. Projeto pedagógico interdisciplinar na e para a formação do fisioterapeuta: dialogando com as diretrizes curriculares. Rev Teoria e Prática da Educação. [on line]. 2009 [citado 12 fevereiro 2013];12(1):17-23. Disponível em: http://periodicos.uem.br/ojs/index.php/TeorPratEduc/article/view/14052/7668

23. Lanzoni GMM, Meirelles BHS. A rede de relações e interações da equipe de saúde na Atenção Básica e implicações para a enfermagem. Acta paul enferm. [on line]. 2012 [citado 12 fevereiro 2013];25(3):464-70. Disponível em: http://www.scielo.br/pdf/ape/v25n3/v25n3a23.pdf

24. Marcondes ALN, Dias R. Características do bullying como um tipo de assédio moral nas organizações. RPCA. [on line]. 2011 [citado 12 fevereiro 2013];5(1):80-7. Disponível em: http://www.uff.br/rpca/Volume\%2011/Assediomoral.pdf 
25. Silva JAM, Peduzzi M. Educação no trabalho na atenção primária à saúde: interfaces entre a educação permanente em saúde e o agir comunicativo. Saúde Soc. [on line]. 2011 [citado 12 fevereiro 2013];20(4):1018-32. Disponível em: http://www.scielo.br/pdf/sausoc/v20n4/18.pdf

26. Paulino VC, Souza PR, Borges CJ. Contribuições da educação permanente em serviço no contexto da Estratégia de Saúde da Família. Itinerarius Reflectionis. [on line]. 2010 [citado 12 fevereiro 2013];2(9):1-11. Disponível em: http://www.revistas.ufg.br/index.php/ritref/article/view/20365/11855 